\title{
Light-Harvesting and Ultrafast Energy Migration in Porphyrin-Based Metal-Organic Frameworks
}

Ho-Jin Son, ${ }^{\dagger, \|}$ Shengye Jin, ${ }^{\ddagger, \|}$ Sameer Patwardhan, ${ }^{\dagger}$ Sander J. Wezenberg, ${ }^{\dagger, \#}$ Nak Cheon Jeong, ${ }^{\dagger, \S}$ Monica So, ${ }^{\dagger}$ Christopher E. Wilmer, ${ }^{\perp}$ Amy A. Sarjeant, ${ }^{\dagger}$ George C. Schatz, ${ }^{\dagger}$ Randall Q. Snurr, ${ }^{\perp}$ Omar K. Farha, ${ }^{* \dagger}$ Gary P. Wiederrecht, ${ }^{*}, *$ and Joseph T. Hupp ${ }^{* \dagger}$

${ }^{\dagger}$ Department of Chemistry, and ${ }^{\perp}$ Chemical and Biological Engineering, Northwestern University, Evanston, Illinois 60208 , United States

${ }^{\ddagger}$ Center for Nanoscale Materials, Argonne National Laboratory, Argonne, Illinois 60439, United States

${ }^{\S}$ Department of Emerging Materials Science, DGIST, Daegu, Korea

\section{Supporting Information}

ABSTRACT: Given that energy (exciton) migration in natural photosynthesis primarily occurs in highly ordered porphyrin-like pigments (chlorophylls), equally highly ordered porphyrin-based metal-organic frameworks (MOFs) might be expected to exhibit similar behavior, thereby facilitating antenna-like light-harvesting and positioning such materials for use in solar energy conversion schemes. Herein, we report the first example of directional, longdistance energy migration within a MOF. Two MOFs, namely FMOF and DA-MOF that are composed of two $\mathrm{Zn}$ (II) porphyrin struts [5,15-dipyridyl-10,20-bis(pentafluorophenyl)porphinato]zinc(II) and [5,15-bis[4-(pyridyl)ethynyl]-10,20-diphenylporphinato]zinc(II), respectively, were investigated. From fluorescence quenching experiments and theoretical calculations, we find that the

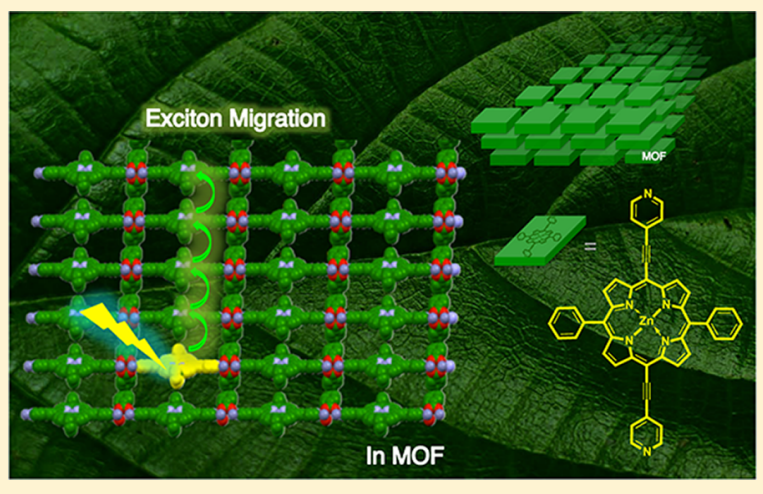
photogenerated exciton migrates over a net distance of up to $\sim 45$ porphyrin struts within its lifetime in DA-MOF (but only $\sim 3$ in F-MOF), with a high anisotropy along a specific direction. The remarkably efficient exciton migration in DA-MOF is attributed to enhanced $\pi$-conjugation through the addition of two acetylene moieties in the porphyrin molecule, which leads to greater Q-band absorption intensity and much faster exciton-hopping (energy transfer between adjacent porphyrin struts). The long distance and directional energy migration in DA-MOF suggests promising applications of this compound or related compounds in solar energy conversion schemes as an efficient light-harvesting and energy-transport component.

\section{INTRODUCTION}

In natural photosynthesis, a core function is the sequential migration of photoinduced energy (exciton) in light-harvesting pigments (i.e., chlorophylls and carotenoids). ${ }^{1}$ Highly ordered structures in antenna are composed of chlorophyll and carotenoid pigments. For example, in purple photosynthetic bacteria, highly symmetric wheel-like supramolecular architectures play an essential role in the absorption of light and the subsequent energy funneling to the reaction centers. ${ }^{2-4}$ These nanometer-sized and three-dimensional structures have motivated chemists to design artificial light-harvesting antenna assemblies such as chromophoric polymers, ${ }^{5}$ dendrimers, ${ }^{6-8}$ covalently linked porphyrin arrays, ${ }^{9}$ and self-assembled supramolecular systems. ${ }^{10-13}$

Recently, metal-organic frameworks (MOFs), which are hybrid materials made from organic linkers and inorganic nodes, have begun to receive attention as alternative compounds for configuration as light-harvesting systems. The high degree of chromophore ordering achievable within crystalline MOFs may provide a basis for systematically relating structure and composition to photon capture and energy transport and delivery. At the same time, the ability with frameworks to position chromophores proximal to each other, yet not in direct physical contact, can prevent self-quenching and deleterious attenuation of exciton lifetimes. A recent study by Lin, Meyer, and co-workers reported facile intracrystal siteto-site energy migration dynamics in $\mathrm{Ru}(\mathrm{II}) / \mathrm{Os}(\mathrm{II})\left(2,2^{\prime}\right.$ bipyridine $)_{3}$-derived MOFs through luminescence quenching measurements. ${ }^{14-17}$ Their work has clearly demonstrated the potential of appropriately designed MOFs to function as lightharvesting and energy-delivery structures. Key to achieving facile migration in the Ru-based MOFs has been long excitedstate lifetimes-a feature absent in natural photosynthetic systems

Received: October 27, 2012

Published: December 18, 2012 
Given that energy migration in natural photosynthesis occurs, in part, through highly ordered porphyrin-like pigments (chlorophylls), we reasoned that crystalline MOFs assembled from photoactive porphyrins might exhibit light-harvesting behavior reminiscent of that of natural photosynthetic systems. However, the use of porphyrin-based MOFs as functional light harvesters (not just absorbers) has not yet been significantly explored. We and our co-workers, as well as others, have previously reported the successful development of porous metalloporphyrin frameworks that can function as catalysts. ${ }^{18-26}$ We have also recently reported that efficient, single-step excitonic energy transfer can be observed in a MOF containing a porphyrin and a complementary chromophore as linkers. ${ }^{27}$ However, important questions, such as how far molecular excitons can migrate, what factors determine the efficiency of the exciton migration, and to what extent excitons can migrate directionally, have not been answered, or even examined, for porphyrinic MOFs. Exploration of these issues is critical for designing and utilizing porphyrin-based MOFs for efficient light-harvesting and energy delivery-for example, to redox-active chemical catalysts. ${ }^{17}$

Herein, we report the design, synthesis and characterization of two MOFs (F-MOF and DA-MOF) constructed from zinc metalated porphyrin $(\mathrm{ZnP})$ molecules. The efficacy of singlet exciton migration in the MOFs is investigated via fluorescence quenching measurements and the migration anisotropy is examined by theoretical calculations. In Figure 1 we show a

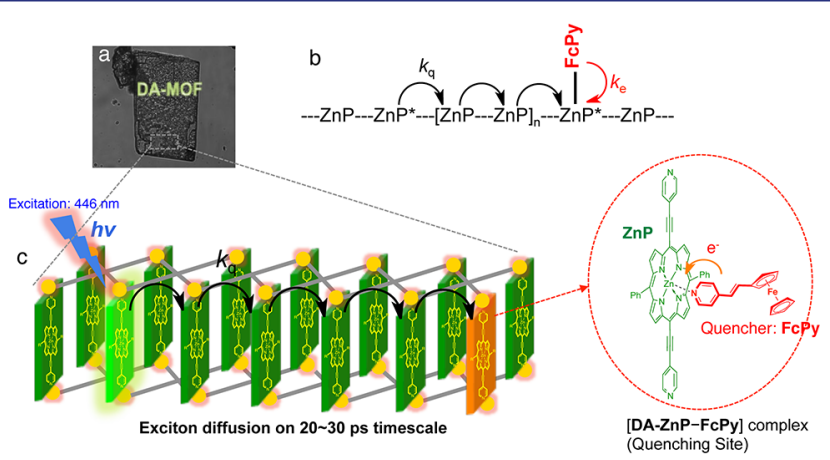

Figure 1. (a) Photograph of a DA-MOF particle from which fluorescence is recorded based on laser excitation at $446 \mathrm{~nm}$. Luminescence quenching measurements are conducted to probe the energy (exciton) migration dynamics. $(b, c)$ Schematic representation of the exciton migration and quenching processes. Upon illumination, the photogenerated exciton can hop between adjacent porphyrin units until it reaches a quenching site. In the red ellipse is an expanded view of the quenching site where the quencher (FCPy) is bound to the porphyrinic $\mathrm{Zn}$ center; the exciton is quenched via electron transfer from $\mathrm{FcPy}$ to the porphyrin entity $(\mathrm{ZnP})$.

photo of a DA-MOF crystallite and a schematic representation of the fluorescence quenching measurements. Illumination of the MOF generates a singlet excition that can migrate by siteto-site (linker-to-linker) hopping within the MOF until it decays (for example, by fluorescence) or is quenched via an encounter with another species. In our experiments, the exciton can be quenched by electron transfer to the excited linker from an axially ligated donor molecule (pyridyl-ferrocene, FcPy). If the donor is present at only a fraction of the available sites, rapid exciton migration results in amplified fluorescence quenching. The efficacy of exciton migration relative to nonreactive exciton decay can be determined by measuring the extent of quenching with various levels of FcPy incorporation. $^{28}$

Based on quenching measurements, we find that the exciton can visit $\sim 90$ porphyrin linkers in DA-MOF and $\sim 6$ in F-MOF within the brief (ca. nanosecond) exciton lifetime. Consistent with the comparatively low symmetry of both the chromophoric and non-chromophoric linkers, detailed computational studies reveal, for both MOFs, that exciton migration is substantially anisotropic. To the extent that exciton transport can be channeled along a single direction, the net distance for energy transport (exciton displacement) can be increased. Directional and long-distance exciton migration that can be rationally modulated is likely to be an important element in the design of functional molecule-derived solar energy conversion systems. The results of the present study provide useful MOF and linker design guidelines for engendering such behavior.

\section{EXPERIMENTAL SECTION}

Structure Characterization of DA-MOF and F-MOF. Several attempts were made to determine the single-crystal X-ray structure of DA-MOF. Although consistent results, based on several syntheses, were obtained for unit cell parameters, we were unable to obtain sufficient data for full structure determination. The unit-cell dimensions $(11.47(3) \times 15.589(3) \times 27.170(6) \AA)$ are appropriate for the proposed framework. With these findings in hand, the hypothetical MOF generator described by Wilmer and co-workers ${ }^{29}$ was used to construct a MOF structure. Three building blocks were used as input: the dipyridylporphyrin linker $\left(\mathrm{DA}-\mathrm{H}_{2} \mathbf{P}\right)$, inorganic $\mathrm{Zn}^{\mathrm{II}}$ paddlewheel nodes, and $\mathrm{Zn}\left(\mathrm{NO}_{3}\right)_{2} \cdot 6 \mathrm{H}_{2} \mathrm{O}, 1,2,4,5$-tetrakis(4carboxyphenyl)benzene (TCPB). In the actual MOF, the porphyrin is likely capable of rotating on its dipyridyl axis; its equilibrium orientation, however, is unknown to us. ${ }^{30}$ For the purpose of generating the hypothetical structure, the plane of the porphyrin was chosen to be at $43.8^{\circ}$ to the plane defined by the $\mathrm{Zn}$ atoms and four of the oxygen atoms of the metal corner. This angle is typical of MOFs with dipyridyl ligands and $\mathrm{Zn}^{\mathrm{II}}$-paddlewheel nodes that have been characterized by single-crystal X-ray diffraction. ${ }^{18}$ The unit cell of the generated structure $(11.59 \times 15.01 \times 27.51 \AA)$ is in an excellent agreement with the experimentally obtained unit cell. Additionally, the simulated powder X-ray diffraction (PXRD) pattern is in excellent agreement with the experimental pattern (see Figure S5). Singlecrystal X-ray structural characterization of F-MOF has been described previously. ${ }^{18}$ PXRD data for F-MOF are shown in Figure S6.

FcPy Doping in MOFs. In non-ligating solvents, pyridine (Py) or FcPy readily binds to porphyrinic $\mathrm{Zn}$ (II) sites. (The binding constants for FcPy and Py to free porphyrin molecules were found to be $4.5 \times$ $10^{4}$ and $1.96 \times 10^{4} \mathrm{M}^{-1}$, respectively, based on the UV-vis titration measurements (See Figure S7).) To incorporate FcPy in a controlled fashion, MOF samples were soaked in various FcPy-containing solutions where the sum of the FcPy and Py concentrations is 0.01 $\mathrm{M}\left(C_{\mathrm{FcPy}}+C_{\mathrm{Py}}=0.01 \mathrm{M}\right)$. Thus, all porphyrinic $\mathrm{Zn}(\mathrm{II})$ sites are ligated. (Note that zinc ions constituting nodes are already coordinatively saturated, so are incapable of functioning as secondary FcPy binding sites.) Since the energies of porphyrin excited-states can be altered slightly via axial coordination of the metal center, this protocol ensures that the energies of the fluorescing states of all porphyrin subunits are essentially identical. Consequently, exciton traps should be absent from these crystalline ensembles. The amount of doped FcPy in the MOF was adjusted by changing the fraction of FcPy in the soaking solution. To quantify the FcPy loading, we determined, via inductively coupled plasma-optical emission spectroscopy (ICP-OES), the mole ratio of Fe to $\mathrm{Zn}$. Estimates of FcPy loading based on the binding constants and on competitive binding were found to agree well with values obtained directly from ICP-OES. (Additionally, the ICP data serve to rule out significant binding to alternative sites such as coordinatively defective MOF node sites.)

Fluorescence Quenching and Lifetime Measurements. MOF fluorescence quenching measurements were carried out with a home- 
built scanning confocal microscope based on an Olympus IX-71 inverted microscope. Samples were excited with a CW laser (Coherent CUBE) at $446 \mathrm{~nm}$. Samples, from which solvent had first been allowed to evaporate, were placed on glass coverslips on a piezo scanner and the excitation beam $(<15 \mathrm{nW})$ was focused through an objective $(60 \times$, NA 1.35, oil immersion, Olympus). The resulting epifluorescence was collected by the same objective. A band-pass filter $(630$ to $730 \mathrm{~nm})$ was placed in the collection path to block stray pump light, with the fluorescence ultimately being detected by a fiber-coupled singlephoton avalanche photodiode (SPAD, Micro Photon Devices). The output of the SPAD was recorded and analyzed by using a timecorrelated single photon counting (TCSPC, PicoQuant) module as counts of photons per second. Low excitation powers $(<15 \mathrm{nW})$ were used so as to avoid exciton-exciton annihilation (self-quenching; see Figure S8 in Supporting Information for the details).

Fluorescence lifetime measurements were carried out with the same confocal microscope. The samples were excited by frequency-doubled pulses from a Ti:sapphire laser (Coherent Micra) at $400 \mathrm{~nm}$ with a repetition rate of $5 \mathrm{MHz}$ (after a pulse picker) and an excitation power of $\sim 300 \mathrm{nW}$. The output of the SPAD detector traveled through the same optical filter and recorded and analyzed by a PicoQuantTCSPC module.

\section{RESULTS AND DISCUSSION}

Initial Characterization. The synthesis of the studied MOFs is presented in Scheme 1. The construction of the

Scheme 1. Synthesis Routes of the Isostructural DA-MOF and F-MOF Compounds and Photographs of Resulting Crystals

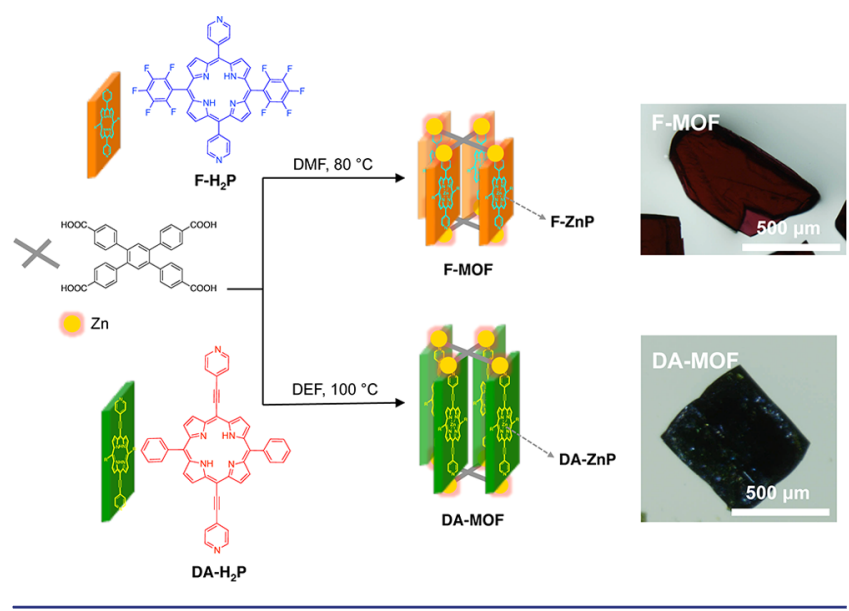

MOFs starts from one of two free-base porphyrin molecules: unsymmetrical [5,15-dipyridyl-10,20-bis(pentafluorophenyl)]porphyrin (designated $\mathbf{F}-\mathbf{H}_{2} \mathbf{P}$ ) or [5,15-di(4-pyridylacetyl)10,20-diphenyl]porphyrin (designated $\mathbf{D A}-\mathbf{H}_{2} \mathbf{P}$ ). These porphyrins were chosen, in part, because they yield non-catenated and fully reactant-accessible active site MOFs, and also because they offer structural and chromophoric similarity to various natural chlorophylls. The synthesis of $\mathbf{F}-\mathbf{H}_{2} \mathbf{P}$ followed a previous report, ${ }^{18}$ while $\mathbf{D A}-\mathbf{H}_{2} \mathbf{P}$ was newly designed for this work. Compared with $\mathbf{F}-\mathbf{H}_{2} \mathbf{P}$, the structure of $\mathbf{D A}-\mathbf{H}_{2} \mathbf{P}$ is extended by addition of acetylene moieties at the 5- and 15positions. The acetylenes enable the pendant pyridine groups to achieve coplanarity with the tetrapyrrole core and thereby extend the molecule's conjugation. In contrast, the phenyl groups at the 10 and 20 positions are not strongly electronically coupled to the porphyrin core. A consequence of the coupling differences is a substantial reduction in electronic symmetry, such that absorption in the Q-band region becomes more strongly allowed than in the electronically more symmetrical F$\mathbf{H}_{2} \mathbf{P}$ molecule. Details of the synthesis of $\mathbf{D A}-\mathbf{H}_{2} \mathbf{P}$ are presented in Scheme S1 in the Supporting Information.

As shown in Scheme 1, the DA- $\mathbf{H}_{2} \mathbf{P}$-based MOF (DAMOF) was synthesized in high yield via standard solvothermal methods in diethylformamide (DEF) using TCPB, and DA$\mathbf{H}_{2} \mathbf{P}$ in the presence of $\mathrm{HNO}_{3}$. The $\mathbf{F}-\mathbf{H}_{2} \mathbf{P}$-based MOF (FMOF) was assembled using a recently reported method. ${ }^{18}$ The MOF nodes consist of pairs of paddlewheel (carboxylate) coordinated $\mathrm{Zn}$ (II) ions that are additionally axially ligated by pyridyl functionalities. Crystallites for both MOFs are plate-like, with lateral dimensions on the order of 100-200 $\mu \mathrm{m}$. As shown in the photographs in Scheme 1, F-MOF and DA-MOF are red and green, respectively.

Shown in Figure 2 are electronic absorption and emission spectra of free F-ZnP and DA-ZnP molecules in dimethylfor-
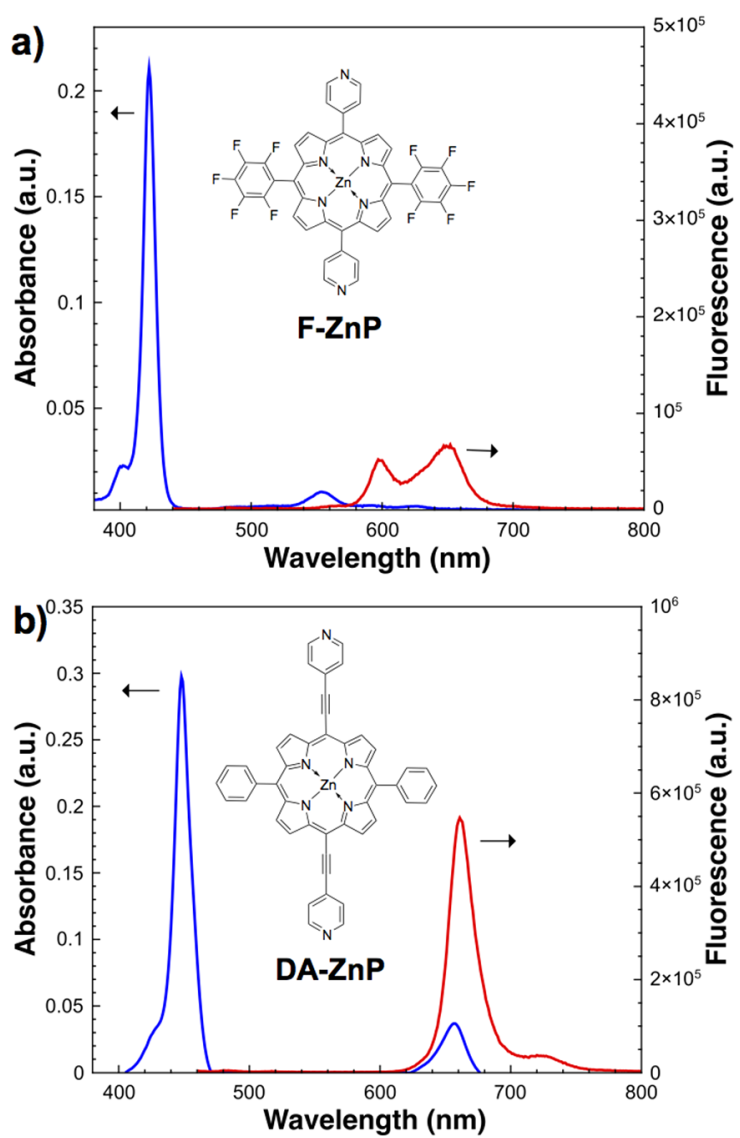

Figure 2. UV-vis absorption (blue) and emission (red) spectra of FZnP (a) and DA-ZnP (b) molecules in DMF. Their chemical structures are shown in the inset.

mamide (DMF). Notably, absorption in the Q-band region (long wavelength region) is substantially red-shifted, as well as intensified, for DA-ZnP, relative to F-ZnP. Specifically, the DA$\mathrm{ZnP}$ absorption coefficient $(\varepsilon)$ is 376000 at $448 \mathrm{~nm}$ and 51 000 at $658 \mathrm{~nm}$, while the photoluminescence quantum yield $\left(\Phi_{\mathrm{DA}}\right)$ is 0.096 . For F-ZnP, $\varepsilon=273000$ at $448 \mathrm{~nm}$ and $\varepsilon=$ 8400 at $555 \mathrm{~nm}$, with $\Phi_{\mathrm{F}}=0.031$. Similar energy and intensity effects, attributable to differences in conjugation and extent of electronic asymmetry, have been observed for related porphyrins. $^{31}$ Importantly, DA-ZnP is characterized by a much larger overlap between emission and absorption spectra than is F-ZnP. As one might anticipate from Förster theory, 
and as discussed further below, the differences in spectral overlap translate into sizable differences in energy migration dynamics.

Thermogravimetric analysis (TGA) data for the assynthesized sample of DA-MOF revealed $35-40 \%$ weight loss due to solvent molecules, thus implying substantial porosity (Figure S9 in the Supporting Information). The two MOFs are similar, with TCPB species coordinated to pairs of $\mathrm{Zn}^{\mathrm{II}}$ ions to form two-dimensional sheets that are pillared by either F-ZnP or DA-ZnP. Figure 3 shows space-filling models

a)
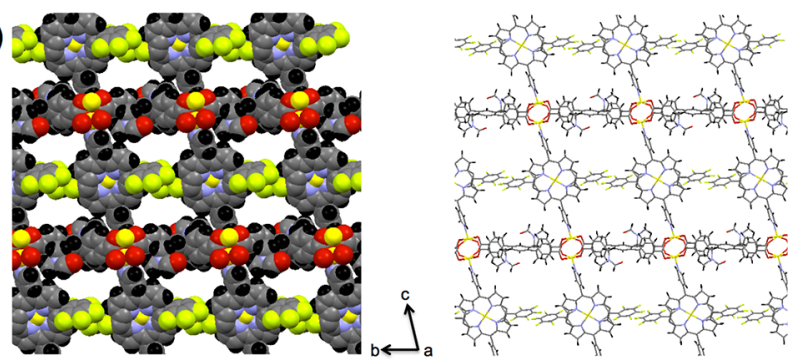

b)

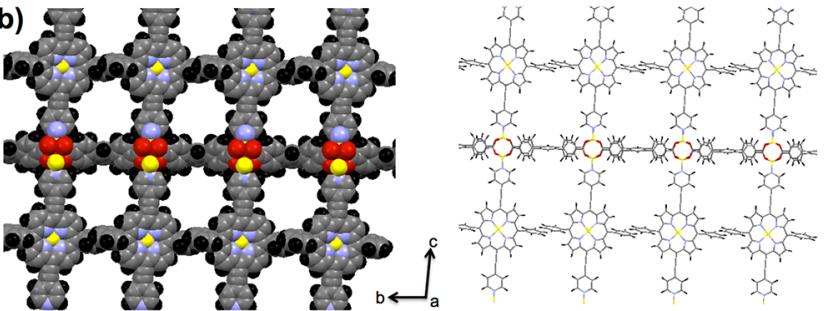

Figure 3. Space-filled (left) and capped stick (right) representations of (a) DA-MOF and (b) F-MOF showing channels in three directions (yellow $=\mathrm{Zn}$, red $=\mathrm{O}$, green $=\mathrm{F}$, blue $=\mathrm{N}$, gray $=\mathrm{C}$, black $=\mathrm{H}$ ). The DA-MOF model was constructed by using the hypothetical MOF generator described by Wilmer and co-workers. ${ }^{29}$.

of the structure of DA-MOF and F-MOF along the various channels. The structures of both reveal large channels in three directions. The $\mathrm{Zn}$-to- $\mathrm{Zn}$ distance between cofacial $\mathrm{ZnPs}$ is $11.60 \AA$ for DA-MOF and $11.57 \AA$ for F-MOF, while the distance between in-plane (collinear) $\mathrm{ZnPs}$ is $27.51 \AA$ for DAMOF and $22.12 \AA$ for F-MOF. DA-MOF features a slightly larger channel height due to the use of a longer porphyrin pillar.

Energy Migration Efficiency in MOFs. Amplified photoluminescence quenching, due to comparatively rapid exciton migration, has been previously reported in MOFs, ${ }^{17}$ porphyrin clay systems ${ }^{32}$ and conjugated polymers. ${ }^{33,34}$ Thus, sufficiently rapid exciton migration within a MOF should enable dramatic fluorescence quenching to be achieved using only small amounts of quencher (see Figure 1). Correlations between the extent of fluorescence quenching and the concentration or amount of quencher can therefore be used to quantify exciton migration efficiency. In previously reported studies, quenching measurements have typically been carried out in the presence of a surrounding solvent, with the quencher present only on the external surfaces of the MOF or conjugated polymer. In this study, FcPy quenchers were incorporated directly inside the MOF structures. ${ }^{35,36}$ A list of DA-MOF and F-MOF samples with different $\mathrm{FcPy}$ doping ratios ranging from $\sim 0.005$ to 1 for fluorescence quenching measurements is provided in Table S1.

Figure $4 \mathrm{a}$ shows the steady-state fluorescence intensity of DA-MOF and F-MOF as a function of FcPy doping ratios. For comparison, a simulated fluorescence quenching curve without exciton migration is also plotted. As shown in Figure 4a, both
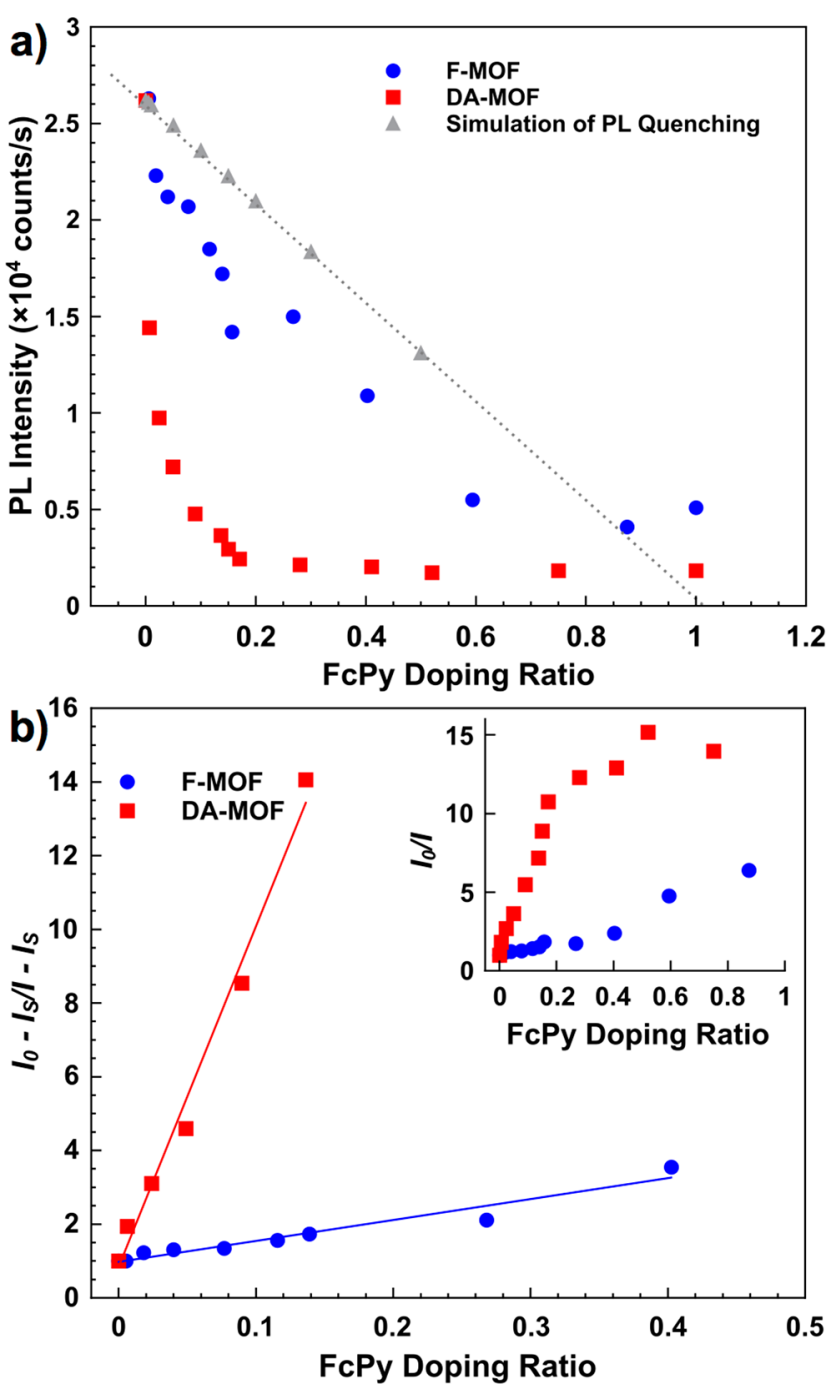

Figure 4. (a) Steady-state fluorescence intensities of F-MOF (closed blue circles) and DA-MOF (closed red squares) as a function of extent of FcPy loading (from 0 to 1 ). The linear gray plot shows the behavior expected when exciton migration is absent and quenching is exceptionally fast relative to the exciton lifetime. (b) The corresponding modified Stern-Volmer plots, based on eq 3. Presented in the inset are Stern-Volmer plots based on eq 2.

F-MOF and DA-MOF exhibit amplified fluorescence quenching, implying significant exciton migration. The amplification behavior, however, is substantially greater for DA-MOF. Remarkably, ca. $50 \%$ of the fluorescence of DA-MOF is quenched when only $0.5 \%$ of the porphryin linkers are ligated by FcPy. For F-MOF, 50\% quenching requires ligation ca. 20\% of the porphyrin units by FcPy to achieve a similar extent of quenching.

The fluorescence quenching in the two MOF samples plateaus after reaching a certain ratio of FcPy, as shown in Figure 4a. This flat level is attributed to the saturation of fluorescence quenching that starts at the ratio of $\sim 0.15$ for DAMOF and $\sim 0.60$ for F-MOF. This phenomenon is similar to the luminescence quenching behavior of phosphorescent MOFs. ${ }^{16}$ The saturation of quenching occurs when the quenching process (electron transfer from FcPy to the excited porphyrin) is not fast enough to completely eliminate the fluorescence. Based on the saturation fluorescence intensity the 
electron transfer rate from FcPy to porphyrin can be estimated according to eq 1 ,

$$
\frac{I_{0}}{I_{\mathrm{s}}}=\frac{\Phi_{0}}{\Phi_{\mathrm{s}}}=1+\tau_{0} k_{\mathrm{e}}
$$

where $I_{0}, \Phi_{0}$, and $\tau_{0}$ are the intrinsic fluorescence intensity, quantum yield, and lifetime without quenchers, respectively; $I_{s}$ and $\Phi_{s}$ are the saturated fluorescence intensity and quantum yield. $k_{\mathrm{e}}$ is the electron transfer (quenching) rate from FcPy to porphyrin, which was estimated to be $\sim 4 \times 10^{9} \mathrm{~s}^{-1}$ in DAMOF and $\sim 1.6 \times 10^{9} \mathrm{~s}^{-1}$ in F-MOF.

To quantify the exciton migration efficiency, quenching data for DA-MOF and F-MOF were analyzed using the SternVolmer (SV) relationship,

$$
\frac{I_{0}}{I_{\mathrm{s}}}=1+K_{\mathrm{SV}}[\mathrm{Q}]
$$

where $I$ is the fluorescence intensity at at a particular level of quencher doping, $K_{\mathrm{SV}}$ is the SV quenching constant, and [Q] is the quencher doping ratio (i.e., the fraction of linkers featuring an axially ligated FCPy molecule). The SV plots, shown in the inset in Figure $4 \mathrm{~b}$, present a roughly linear dependence in the region with FcPy ratio less than 0.16 for DA-MOF and less than 0.6 for F-MOF, but deviate from this dependence and reach a flat level due to the saturation of fluorescence quenching at higher $\mathbf{F c P y}$ ratios. The presence of residual fluorescence at the saturation level reduces the value of fluorescence amplification $\left(I_{0} / I\right)$, and therefore we modified the SV relationship by subtracting the saturated fluorescence intensity $I_{s}$, as in eq 3 :

$$
\frac{I_{0}-I_{\mathrm{s}}}{I-I_{\mathrm{s}}}=1+K_{\mathrm{SV}}[\mathrm{Q}]
$$

Modified SV curves, with only the linear section plotted for the two MOFs, are shown in Figure $4 \mathrm{~b}$. Since the quencher is directly doped into the MOF structure, the fluorescence quenching should not follow a diffusional controlled mechanism, but a quenching mechanism with $K_{\mathrm{SV}}=N_{\mathrm{t}}$ where $N_{\mathrm{t}}$ is the number of the porphyrin sites that the exciton visits within its lifetime. Fits of the plots with eq 3 yield $K_{\mathrm{SV}}$ values of 91 for DA-MOF and 5.6 for F-MOF, indicating that an exciton visits, on average, about 90 distinct porphyrin sites in DA-MOF and 6 sites in F-MOF within its lifetime.

The above quenching measurements indicate that the energy migration efficiency in DA-MOF is much higher than that in FMOF. As discussed further below, in the low-power limit the fluorescence lifetimes of the two photoexcited MOFs are similar; consequently, the differences in migration efficiency can be largely understood in terms of differences in the dynamics of migration. Energy migration in MOFs or conjugated polymers involves sequential hopping of excitons between adjacent chromophores, the rate of which (for singlet excitons) is describable by the Förster energy transfer model ${ }^{17,37}$ (albeit, sometimes with extensions beyond the point-dipole model; see Supporting Information for details of the approach used here). The hopping rate for singlet excitons depends on the spectral overlap between emission and lowest-energy (singlet) absorption bands of the molecular building blocks, and on the separation distance $(r)$ between the energy donor and acceptor. In the point-dipole limit, the hopping rate scales as $1 /$ $r^{6}$.
Energy Migration Anisotropy in MOFs. The exciton migration anisotropy in DA-MOF and F-MOF can be evaluated through theoretical calculations. The four possible exciton directions (from A to B, C, D, or E) with corresponding intermolecular $(\mathrm{Zn}-\mathrm{Zn})$ distances have been depicted in Figure 5 for both MOFs. Nominally one would expect the

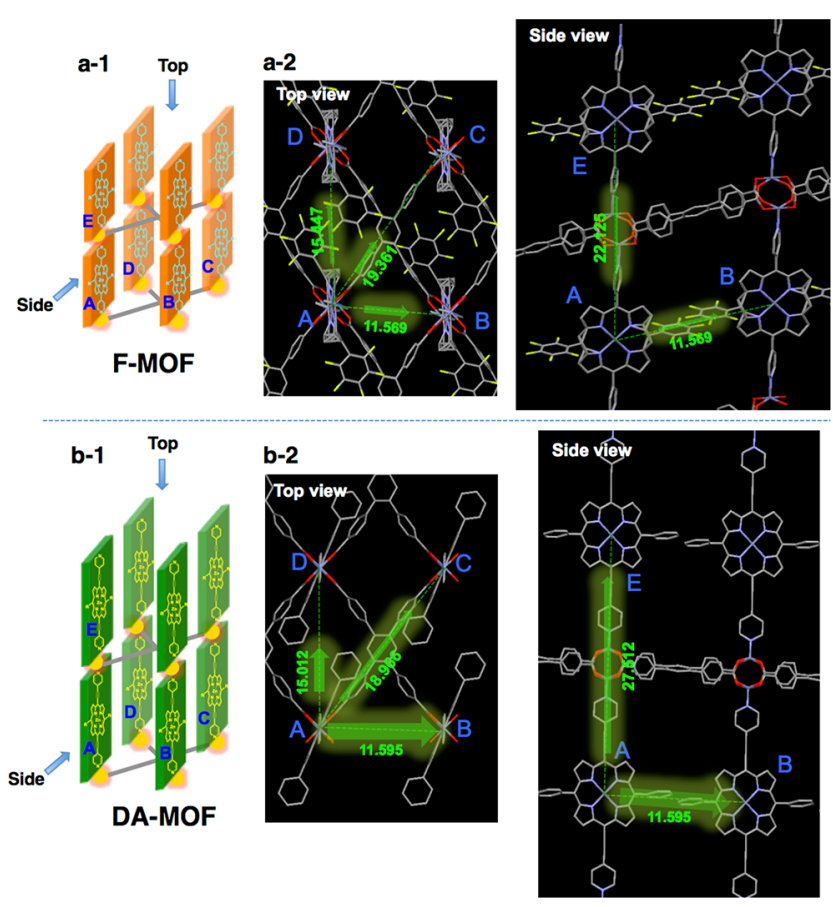

Figure 5. Schematic presentations of the structural configurations for F-MOF (a-1) and DA-MOF (b-1). The letters on the porphyrin blocks indicates the 4 nearest neighbors $\mathrm{B}, \mathrm{C}, \mathrm{D}$ and $\mathrm{E}$ around $\mathrm{A}$. The capped stick representations of the crystal structure of F-MOF (a-2) and DA-MOF (b-2) with arrows indicating the four energy transfer directions from $\mathrm{A}$ to $\mathrm{B}, \mathrm{C}, \mathrm{D}$ and $\mathrm{E}$ between the nearest neighboring porphyrin blocks. The center-to-center distances between pairs of porphyrin molecules along each direction are also indicated beside the arrows.

direction with the highest exciton-hopping rate to be the preferred exciton migrating direction. The rate of exciton hopping between adjacent porphyrin linkers along each direction is calculated using the Förster energy transfer equation (eq 4).

$$
k_{\mathrm{EnT}}=\frac{2 \pi}{\hbar} J^{2} \mathrm{OI}
$$

According to this equation the rate constant $\left(k_{\mathrm{EnT}}\right)$ for exciton hopping is governed by two key parameters: the overlap integral (OI) between the normalized absorption and emission spectra and the exciton coupling $(J)$.

The overlap integrals for DA-MOF and F-MOF, calculated from the corresponding experimental absorption and fluorescence spectra of non-aggregated monomers in solution, are 0.57 and $0.19 \mathrm{eV}^{-1}$, respectively. Two low-lying excited states (ES1 and ES2) overlap in the Q-band region and both excited states will participate in the energy transfer process. The oscillator strength (os) of the Q-band, which is indirectly related to the exciton coupling of eq 4 (os $\left.\propto \mu^{2} \propto J\right)$, is higher for the porphyrin strut in DA-MOF (0.21) than for the strut in F-MOF (0.06). 
Exciton couplings were evaluated using atomic transition densities. $^{38-40}$ (See Supporting Information for details of the approach used.) When chromophores are poorly approximated by spheres, this approach yields much more reliable estimates than does a point-dipole approximation. Couplings associated with the exciton transitions ES1-ES1, ES2-ES2, and ES1-ES2 (cross) have been calculated. Because internal relaxation is fast, coupling involving higher energy states is unimportant. The effects of disorder of porphyrin units around the dipyridyl axes have been taken into account by considering and then summing (with equal population weighting) contributions from each of several rotational conformations. The calculated exciton hopping rates along different directions are assembled in Table 1. In DA-MOF the hopping rates for the second excited

Table 1. Calculated Förster Energy Transfer (Exciton Hopping) Rate Constants $k_{\mathrm{EnT}}$ along the Four Directions $\mathrm{AB}, \mathrm{AD}, \mathrm{AE}$, and $\mathrm{AC}$ in F-MOF and DA-MOF Based on Eq $4^{a}$

\begin{tabular}{|c|c|c|c|c|}
\hline & ES1 $\left(10^{9} \mathrm{~s}^{-1}\right)$ & $\operatorname{ES} 2\left(10^{9} \mathrm{~s}^{-1}\right)$ & $\operatorname{ES} 3\left(10^{9} \mathrm{~s}^{-1}\right)$ & $\begin{array}{c}\mathrm{ES} 1+\mathrm{ES} 2+\mathrm{ES} 3 \\
\left(10^{9} \mathrm{~s}^{-1}\right)\end{array}$ \\
\hline \multicolumn{5}{|c|}{ F-MOF } \\
\hline $\mathrm{AB}$ & 0.4 & 0.28 & 6.70 & 7.4 \\
\hline $\mathrm{AD}$ & 7.2 & 0.47 & 2.60 & 10.2 \\
\hline $\mathrm{AE}$ & 0.5 & 0.07 & 0.02 & 0.6 \\
\hline $\mathrm{AC}$ & 1.4 & 0.01 & 0.01 & 1.5 \\
\hline \multicolumn{5}{|c|}{ DA-MOF } \\
\hline $\mathrm{AB}$ & 99 & 4.09 & 0.04 & 103 \\
\hline $\mathrm{AD}$ & 30 & 2.54 & 0.08 & 33 \\
\hline $\mathrm{AE}$ & 39 & 0.08 & 0.01 & 39 \\
\hline $\mathrm{AC}$ & 11 & 0.79 & 0.00 & 12 \\
\hline
\end{tabular}

${ }^{a}$ Rates of energy transfer from ES1 to ES1, ES2 to ES2, and ES1 to ES2 have been considered. Each rate is averaged over 25 rotational conformations (the rotation angles of $-30^{\circ},-15^{\circ}, 0^{\circ}, 15^{\circ}$, and $30^{\circ}$ around the bipyridyl axis of a porphyrin molecule).

state (ES2) are comparatively small; they are negligible for inter-excited-state (cross) transfer. The rate of energy transfer is greatest along the $\mathrm{AB}$ direction, exceeding by ca. 3- to 9-fold the total rates in each of the other directions. Qualitatively this is expected, since the distance between nearest neighbor chromophores is smallest in the $\mathrm{AB}$ direction. Within F-MOF, however, energy transfer rate is greatest in the $\mathrm{AD}$ direction, an effect that appears to arise from framework tilting.

Notably, the fastest rate in Table 1 for DA-MOF is about an order of magnitude larger than the fastest for F-MOF. This difference is attributed to the increased conjugation along the dipyridyl axis of the porphyrin strut in DA-MOF compared to F-MOF. The enhanced conjugation, in turn, leads to decreased electronic symmetry, increased Q-band oscillator strength (increased $J$ values), and better absorption/emission spectral overlap (Figure 2).

In addition to quantifying directional dynamics, we also are interested in obtaining estimates of exciton transport distance in various directions. As a starting point, we considered the case of strictly one-dimensional transport. If an exciton in DA-MOF visits 90 distinct fluorophores, its absolute displacement at the end of its lifetime will, on average, be 45 linkers (fluorophores). Because each successive hop can place the exciton either one site (linker) closer or one site further from its point of origin (i.e., the linker that absorbed the photon that created the exciton), the total number of hops made by the exciton will, on average, be $45^{2}=2025$. For F-MOF, the total number of hops will be $2.8^{2}$ or about 8 .

The average number of hops (both forward and backward) made along each of four possible directions $(\mathrm{AB}, \mathrm{AC}, \mathrm{AD}$, and $\mathrm{AE})$ can be estimated from the hopping rate in a designated direction relative to the sum of hopping rates in all possible directions, times the total number of hops made. For example, for DA-MOF the fraction of hops occurring along $A B$ is $k_{\mathrm{EnT}}(\mathrm{AB}) /\left\{k_{\mathrm{EnT}}(\mathrm{AB})+k_{\mathrm{EnT}}(\mathrm{AD})+k_{\mathrm{EnT}}(\mathrm{AE})+k_{\mathrm{EnT}}(\mathrm{AC})\right\}=$ 0.55 . The absolute number of $A B$-directed hops is $0.55 \times 2025$ $=1114$ hops. The root-mean-square displacement of the exciton due to hops along the $\mathrm{AB}$ direction is $(1114)^{1 / 2}=33$ hops. Table 2 lists the full set of estimates of directional

Table 2. Net Exciton Migration Displacement in the Number of Hops and Distances ( $\AA$ ) along the Four Directions AB, $\mathrm{AD}, \mathrm{AE}$, and $\mathrm{AC}$ in F-MOF and DA-MOF

\begin{tabular}{lccc} 
& $\begin{array}{c}\text { fraction of } \\
\text { hops }\end{array}$ & $\begin{array}{c}\text { net displacement in } \\
\text { hops }\end{array}$ & $\begin{array}{c}\text { net displacement in distance } \\
(\AA)\end{array}$ \\
$\mathrm{AB}$ & 0.38 & 1.8 & 21 \\
$\mathrm{AD}$ & 0.52 & 2.2 & 34 \\
$\mathrm{AE}$ & 0.03 & 0.5 & 11 \\
$\mathrm{AC}$ & 0.07 & 0.8 & 15 \\
$\mathrm{AB}^{\prime}$ & - & 2.0 & 23 \\
$\mathrm{AD}$ & - & 2.3 & 36 \\
& & DA-MOF & \\
$\mathrm{AB}$ & 0.55 & 33 & 380 \\
$\mathrm{AD}$ & 0.18 & 19 & 290 \\
$\mathrm{AE}$ & 0.21 & 21 & 580 \\
$\mathrm{AC}^{\prime}$ & 0.06 & 11 & 210 \\
$\mathrm{AB}^{\prime}$ & - & 35 & 410 \\
$\mathrm{AD}^{\prime}$ & - & 22 & 330 \\
\hline
\end{tabular}

displacements for excitons in both MOFs. As one expects based on estimates of hopping rate, the displacements, in terms of numbers of hops, are significantly anisotropic. If we recognize that a hop in the AC direction is equivalent to one hop in the $\mathrm{AB}$ direction + one hop in the $\mathrm{AD}$ direction, then the $\mathrm{AB}$ - and $\mathrm{AD}$-directed displacements will be slightly greater than obtained otherwise. The modified displacement results are listed in Table 2 as $\mathrm{AB}^{\prime}$ and $\mathrm{AD}^{\prime}$ displacements.

It is important to recall that the distance covered in a hop depends strongly on the direction of the hop. For example, for DA-MOF the step sizes in the $\mathrm{AB}$ and $\mathrm{AE}$ directions are ca. 11 and $27 \AA$, respectively. As a consequence, and as shown in Table 2, the exciton displacement distance in DA-MOF is greatest in the $\mathrm{AE}$ direction (ca. $580 \AA$ ) even though the number of exciton hops along the AE direction is less than $40 \%$ of the number made along the $\mathrm{AB}$ direction. The $\mathrm{AE}$ direction is the direction defined by porphyrin-porphyrin collinear alignment. In contrast, for F-MOF the largest displacement distance is attained along the $\mathrm{AD}$ direction, i.e. in a direction parallel to the plane defined by the non-chromophoric linker.

Given that the fluorescence lifetimes of DA-MOF and FMOF in the absence of FcPy are 2.9 and 4.9 ns (see Figure S10 and Table S2 in Supporting Information for the lifetime measurements), respectively, the estimated exciton hopping times are $\sim 2900 / 2025=1.4$ ps for DA-MOF and $\sim 4900 / 7.8=$ 620 ps for F-MOF, respectively. The exciton hopping times derived from the experimental data are broadly consistent with those listed in Table 1, suggesting that the Förster energy 
transfer model (eq 4) as implemented here (see Supporting Information for details of calculations of $J$ ) is reasonably reliable for estimating the exciton hopping rate and its migration anisotropy.

\section{CONCLUSIONS}

We have studied molecular-exciton migration within two $\mathrm{Zn}$ porphyrin-based MOFs (DA-MOF and F-MOF). In both cases, transport is significantly anisotropic. Because of the strong sensitivity of coupling constants ( $J$ values) to separation distance, modest increases in the $\mathrm{AB}$ - and AC-defining dimensions of the non-chromophoric, tetra-carboxylate linker should lead to predominantly AE-directed transport of excitons in DA-MOF. Conversely, shrinking the dimensions of the nonchromophoric linker, and thereby positioning chromophores closer in a lateral, but not vertical, sense, should disfavor transport along the $\mathrm{AE}$ direction (i.e., the chromophore end-toend direction).

Eliminating $\mathrm{Zn}$ (II) from the porphyrin centers would likely increase exciton lifetimes. All else being equal, a 4-fold increase in singlet exciton lifetime would yield 2 -fold increases in exciton displacements. In reality, elimination of the zinc centers will reduce the symmetry of each of the porphyrins and split the $Q$ bands. In all likelihood, the splitting will alter spectral overlaps and thereby further affect hopping dynamics and transport distances. If the goal is to increase exciton migration distances, a more effective strategy would likely be to further increase the electronic asymmetry of the porphyrin linker in DA-MOF, thereby increasing oscillator strengths in the Q-band region and boosting both spectral overlap and coupling constants. Porphyrin dimerization (via ethyne or butadiyne connectors) is known to elicit such increases in Q-band absorption intensity. ${ }^{41-43}$ Alternatively, triplet exciton migration might prove effective. Often the primary decay pathway from fluorescent excited states of zinc porphyrins is intersystem conversion to a triplet excited state. In the absence of molecular oxygen, such states often persist for several orders of magnitude longer than the corresponding singlet states. Although rates of triplet exciton migration are likely to be many times slower than singlet migration rates, the enhanced exciton lifetime could conceivably result in longer displacement distances. Regardless, because the triplet exciton is formed at the final destination of the singlet exciton, rather than at the site of photon absorption, the exciton displacement distance (singlet + triplet contributions) should exceed the displacement attained by singlet migration alone.

Returning to DA-MOF and F-MOF, we find that the average numbers of chromophore-to-chromophore hops by singlet excitons are about 2000 and 8 , respectively. These translate into a roughly 16 -fold difference in the number of potential fluorophores visited by excitons in the two MOFs. The differences arise from the reduced electronic symmetry and enhanced linear conjugation obtained with DA-MOF by incorporating an acetylene unit between the tetrapyrrole core and each of the two pyridyl anchoring groups of the porphyrinic linker. These differences, in turn, engender greater Q-band oscillator strength and better absorption/emission spectral overlap, culminating, for DA-MOF in greater dipolar coupling and a larger overlap integral.

The substantial distances and sizable anisotropy of singlet exciton migration in DA-MOF suggest that it or related framework compounds could prove useful as antenna-type light-harvesters in solar energy conversion systems.

\section{ASSOCIATED CONTENT}

\section{Supporting Information}

Experimental details, synthetic procedures and characterization (NMR and mass spectra) of chromophores, PXRD of samples, results of fluorescence decay, TGA, and detailed descriptions of the theoretical calculations. This material is available free of charge via the Internet at http://pubs.acs.org.

\section{AUTHOR INFORMATION}

\section{Corresponding Author}

o-farha@northwestern.edu; wiederrecht@anl.gov; j-hupp@ northwestern.edu

\section{Present Address}

\#Laboratory of Organic Chemistry, Department of Chemistry and Applied Biosciences, ETH Zurich, CH-8093 Zurich, Switzerland

\section{Author Contributions}

${ }^{\|}$H.-J.S. and S.J. contributed equally.

\section{Notes}

The authors declare no competing financial interest.

\section{ACKNOWLEDGMENTS}

S.P. thanks Dr. F. C. Grozema from Delft University of Technology for providing assistance with semiempirical INDO/CIS calculations. G.C.S. gratefully acknowledges the AFOSR (grant no. AFOSR FA9550-11-1-0275) for support of computational work. J.T.H. gratefully acknowledges support at Northwestern from the U.S. Department of Energy, Office of Science, Basic Energy Sciences, through grant no. DE-FG0287ER13808 for studies of molecular light-harvester and MOF synthesis, MOF characterization, and quencher incorporation. S.J.W. thanks the Spanish Ministry of Education for a predoctoral FPU fellowship. S.J. acknowledges support at Argonne from the ANSER Center, an Energy Frontier Research Center funded by the U.S. Department of Energy, Office of Science, Office of Basic Energy Sciences, under award number DESC0001059 (fluorescence spectroscopy). Use of the Center for Nanoscale Materials, and support of G.P.W., is funded by the U.S. Department of Energy, Office of Science, Office of Basic Energy Sciences through contract no. DE-AC02- 06CH11357.

\section{REFERENCES}

(1) Scholes, G. D.; Fleming, G. R.; Olaya-Castro, A.; van Grondelle, R. Nat. Chem. 2011, 3, 763.

(2) Barber, J.; Andersson, B. Nature 1994, 370, 31.

(3) Kuhlbrandt, W.; Wang, D. N.; Fujiyoshi, Y. Nature 1994, 367, 614.

(4) McDermott, G.; Prince, S. M.; Freer, A. A.; HawthornthwaiteLawless, A. M.; Papiz, M. Z.; Cogdell, R. J.; Isaacs, N. W. Nature 1995, 374, 517.

(5) Webber, S. E. Chem. Rev. 1990, 90, 1469.

(6) Sykora, M.; Maxwell, K. A.; DeSimone, J. M.; Meyer, T. J. Proc. Natl. Acad. Sci. U.S.A. 2000, 97, 7687.

(7) Fréchet, J. M. J. J. Polym. Sci., Part A: Polym. Chem. 2003, 41, 3713.

(8) Balzani, V.; Bergamini, G.; Ceroni, P.; Vögtle, F. Coord. Chem. Rev. 2007, 251, 525.

(9) Aratani, N.; Kim, D.; Osuka, A. Acc. Chem. Res. 2009, 42, 1922.

(10) Li, X.; Sinks, L. E.; Rybtchinski, B.; Wasielewski, M. R. J. Am. Chem. Soc. 2004, 126, 10810.

(11) Kelley, R. F.; Lee, S. J.; Wilson, T. M.; Nakamura, Y.; Tiede, D. M.; Osuka, A.; Hupp, J. T.; Wasielewski, M. R. J. Am. Chem. Soc. 2008, $130,4277$. 
(12) Sengupta, S.; Ebeling, D.; Patwardhan, S.; Zhang, X.; von Berlepsch, H.; Böttcher, C.; Stepanenko, V.; Uemura, S.; Hentschel, C.; Fuchs, H.; Grozema, F. C.; Siebbeles, L. D. A.; Holzwarth, A. R.; Chi, L.; Würthner, F. Angew. Chem., Int. Ed. 2012, 51, 6378.

(13) Patwardhan, S.; Sengupta, S.; Siebbeles, L. D. A.; Würthner, F.; Grozema, F. C. J. Am. Chem. Soc. 2012, 134, 16147.

(14) Kent, C. A.; Mehl, B. P.; Ma, L.; Papanikolas, J. M.; Meyer, T. J.; Lin, W. J. J. Am. Chem. Soc. 2010, 132, 12767.

(15) Wang, C.; Lin, W. J. Am. Chem. Soc. 2011, 133, 4232.

(16) Kent, C. A.; Liu, D.; Ma, L.; Papanikolas, J. M.; Meyer, T. J.; Lin,

W. J. J. Am. Chem. Soc. 2011, 133, 12940.

(17) Kent, C. A.; Liu, D.; Meyer, T. J.; Lin, W. J. J. Am. Chem. Soc. 2012, 134, 3991.

(18) Shultz, A. M.; Farha, O. K.; Hupp, J. T.; Nguyen, S. T. J. Am. Chem. Soc. 2009, 131, 4204.

(19) Farha, O. K.; Shultz, A. M.; Sargeant, A. A.; Nguyen, S. T.; Hupp, J. T. J. Am. Chem. Soc. 2011, 133, 5652.

(20) Zhang, Z.; Zhang, L.; Wojtas, L.; Nugent, P.; Eddaoudi, M.; Zaworotko, M. J. J. Am. Chem. Soc. 2012, 134, 924.

(21) Zhang, Z.; Zhang, L.; Wojtas, L.; Eddaoudi, M.; Zaworotko, M. J. J. Am. Chem. Soc. 2012, 134, 928.

(22) Wang, X.-S.; Meng, L.; Cheng, Q.; Kim, C.; Wojtas, L.; Chrzanowski, M.; Chen, Y.-S.; Zhang, X. P.; Ma, S. J. Am. Chem. Soc. 2011, 133, 16322.

(23) Suslick, K. S.; Bhyrappa, P.; Chou, J.-H.; Kosal, M. E.; Nakagaki, S.; Smithenry, D. W.; Wilson, S. R. Acc. Chem. Res. 2005, 38, 283.

(24) Feng, D.; Gu, Z.-Y.; Li, J.-R.; Jiang, H.-L.; Wei, Z.; Zhou, H.-C. Angew. Chem., Int. Ed. 2012, 51, 10307.

(25) Morris, W.; Volosskiy, B.; Demir, S.; Gándara, F.; McGrier, P. L.; Furukawa, H.; Cascio, D.; Stoddart, J. F.; Yaghi, O. M. Inorg. Chem. 2012, 51, 6443.

(26) Burnett, B. J.; Barron, P. M.; Choe, W. CrystEngComm 2012, 14, 3839.

(27) Lee, C. Y.; Farha, O. K.; Hong, B. J.; Sargeant, A. A.; Nguyen, S. T.; Hupp, J. T. J. Am. Chem. Soc. 2011, 133, 15858.

(28) To ensure that the experiments were not complicated by small differences in excited-state energy for zinc(II)-ligated versus nonligated porphyrin linkers, pyridine was bound (axially) to all of the $\mathrm{ZnP}$ units that were not coordinated by FcPy.

(29) Wilmer, C. E.; Leaf, M.; Lee, C. Y.; Farha, O. K.; Hauser, B. G.; Hupp, J. T.; Snurr, R. Q. Nat. Chem. 2012, 4, 83.

(30) Gould, S. L.; Tranchemontagne, D.; Yaghi, O. M.; GarciaGaribay, M. A. J. Am. Chem. Soc. 2008, 130, 3246.

(31) LeCours, S. M.; DiMagno, S. G.; Therien, M. J. J. Am. Chem. Soc. 1996, 118, 11854.

(32) Ishida, Y.; Shimada, T.; Masui, D.; Tachibana, H.; Inoue, H.; Takagi, S. J. Am. Chem. Soc. 2011, 133, 14280.

(33) Thomas, S. W., III; Joly, G. D.; Swager, T. M. Chem. Rev. 2007, 107, 1339.

(34) Liu, Y.; Ogawa, K.; Schanze, K. S. J. Photochem. Photobiol. CPhotochem. Rev. 2009, 10, 173.

(35) Mata, J.; Uriel, S.; Peris, E.; Llusar, R.; Houbrechts, S.; Persoons, A. J. Organomet. Chem. 1998, 562, 197.

(36) Bucher, C.; Devillers, C. H.; Moutet, J.-C.; Royal, G.; SaintAman, E. Coord. Chem. Rev. 2009, 253, 21.

(37) Müller, J. G.; Atas, E.; Tan, C.; Schanze, K. S.; Kleiman, V. D. J. Am. Chem. Soc. 2006, 128, 4007.

(38) Patwardhan, S.; Tonzani, S.; Lewis, F. D.; Siebbeles, L. D. A.; Schatz, G. C.; Grozema, F. C. J. Phys. Chem. B 2012, 116, 11447.

(39) Beenken, W. J. D.; Pullerits, T. J. Chem. Phys. 2004, 120, 2490.

(40) Patwardhan, S.; Sengupta, S.; Würthner, F.; Siebbeles, L. D. A.; Grozema, F. J. Phys. Chem. C 2010, 114, 20834.

(41) Angiolillo, P. J.; Uyeda, H. T.; Duncan, T. V.; Therien, M. J. J. Phys. Chem. B 2004, 108, 11893.

(42) Zhang, T.-G.; Zhao, Y.; Asselberghs, I.; Persoons, A.; Clays, K.;

Therien, M. J. J. Am. Chem. Soc. 2005, 127, 9710.

(43) Lovett, J. E.; Hoffmann, M.; Cnossen, A.; Shutter, A. T. J.; Hogben, H. J.; Warren, J. E.; Pascu, S. I.; Kay, C. W. M.; Timmel, C. R.; Anderson, H. L. J. Am. Chem. Soc. 2009, 131, 13852. 